\title{
Polarity-Regulating Kinase Partitioning-Defective 1/Microtubule Affinity-Regulating Kinase 2 Negatively Regulates Development of Dendrites on Hippocampal Neurons
}

\author{
Takeshi Terabayashi, ${ }^{1}$ Tomohiko J. Itoh, ${ }^{2}$ Hideki Yamaguchi, ${ }^{3}$ Yuta Yoshimura, ${ }^{1}$ Yosuke Funato, ${ }^{1}$ Shigeo Ohno, ${ }^{4}$ and

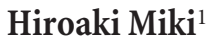 \\ ${ }^{1}$ Laboratory of Intracellular Signaling, Institute for Protein Research, Osaka University, Suita, Osaka 565-0871, Japan, ${ }^{2}$ Division of Biological Sciences, \\ Graduate School of Science, Nagoya University, Chikusa-ku, Nagoya 464-8602, Japan, ${ }^{3}$ Laboratory of Genome and Biosignal, Tokyo University of Pharmacy \\ and Life Science, Hachioji, Tokyo 192-0392, Japan, and ${ }^{4}$ Department of Molecular Biology, Yokohama City University School of Medicine, Kanazawa-ku, \\ Yokohama 236-0004, Japan
}

Neurons are highly polarized cells that possess two morphologically and functionally different types of protrusions, axons and dendrites, that function in the transmission and reception of neural signals, respectively. A great deal of attention has been paid to the specification and guidance of axons, but the mechanism of dendrite development remains mostly unknown. We report here that a polarity-regulating kinase, partitioning-defective 1 (Par1b)/microtubule affinity-regulating kinase 2 (MARK2), specifically regulates development of dendrites in hippocampal neurons. Ectopic expression of Par1b/MARK2 shortens the length and decreases branching of dendrites without significant effects on axons. Knockdown of endogenous Par1b/MARK2 by RNA interference stimulates dendrite development. Wnt stimulation and Dishevelled expression, both of which are known to induce dendrite development, induced recruitment of Par1b/MARK2 to the membrane fraction. Expression of a Par1b/MARK2 mutant, that contains a myristoylation signal and accumulates exclusively in membranes, does not affect dendrite development. In addition, Par1b/MARK2 efficiently phosphorylated MAP2, which is localized mainly in dendrites. These results indicate that Par1b/MARK2 negatively regulates dendrite development through phosphorylation of MAP2.

Key words: dendrite; Wnt; Par1b/MARK2; dishevelled; translocation; MAP2

\section{Introduction}

Partitioning-defective 1 (Par1) is a serine/threonine kinase originally identified as a polarity-regulating gene product in Caenorhabditis elegans (Guo and Kemphues, 1995). In mammals, there are several Par1-related proteins (Tassan and Le Goff, 2004), and Parlb is the best characterized. The polarity-regulating function of Par1 is evolutionarily conserved, and it has been shown that Par1 is important in the regulation of apical-basolateral polarity of epithelial cells (Bohm et al., 1997; Cohen et al., 2004a,b; Hurov et al., 2004; Suzuki et al., 2004).

Illenberger et al. (1996) discovered the serine/threonine kinase microtubule affinity regulating kinase (MARK) as an ortholog of C. elegans Par1, which phosphorylates several microtubule-binding proteins (MAPs). In vitro, MARK phos-

\footnotetext{
Received Dec. 3, 2006; revised 0ct. 11, 2007; accepted 0ct. 11, 2007.

This work was supported in part by a Grant-in-Aid for Scientific Research from the Ministry of Education, Science and Culture of Japan. We thankS. I. Ohmi, J. Inagaki, H. Kosako, K. Nishibashi, and O. Kobayashi for helpful advice and support.

Correspondence should be addressed to Hiroaki Miki, Laboratory of Intracellular Signaling, Institute for Protein Research, 0saka University, 3-2 Yamadaoka, Suita, 0saka 565-0871, Japan. E-mail: hmiki@protein.osaka-u.ac.jp. D0I:10.1523/JNEUROSCI.3986-07.2007

Copyright $\odot 2007$ Society for Neuroscience $\quad$ 0270-6474/07/2713098-10\$15.00/0
}

phorylates MAPs, causing them to detach from microtubules, and destabilizes the microtubules (Drewes et al., 1997). Indeed, overexpression of MARK in Chinese hamster ovary (CHO) cells induces destruction of the microtubule cytoskeleton (Illenberger et al., 1996).

In mammalian epithelial cells, Par1/MARK localizes predominantly at basolateral membranes and is excluded from apical areas (Bohm et al., 1997). Par1/MARK is phosphorylated by atypical protein kinase C (aPKC) (Hurov et al., 2004; Kusakabe and Nishida, 2004; Suzuki et al., 2004), and then moves to the cytosol. aPKC localizes at the tight junctions, and thus this phosphorylation results in the exclusion of Parl/MARK from apical areas. However, it remains controversial whether cytosolic Par1/MARK is catalytically and biologically active.

A previous report indicated that Par1b/MARK2 is essential for axon specification of hippocampal neurons in culture (Chen et al., 2006). Hippocampal neurons have been used routinely for analyses of axon-dendrite polarity formation and maintenance. In the axon-specification process, it has been shown that several molecules, such as CRMP2 (Inagaki et al., 2001), play critical roles (Arimura and Kaibuchi, 2005; Wiggin et al., 2005). Overexpression of Par1b/MARK2 induced formation of neurons with- 
out axons and knockdown of endogenous Par1b/MARK2 resulted in neurons with multiple axons (Chen et al., 2006). These results clearly indicate that Par1b/MARK2 acts negatively in the axon-specification process.

Accumulating data suggest the importance of Wnt signaling in dendrite development after axon specification. Stimulation of cells with Wnt or Wnt antagonist results in elongation or retraction of dendrites, respectively (Rosso et al., 2005). Interestingly, such dramatic effects were observed specifically in dendrites. It was also reported that Wnt is secreted by neurons and that depolarizing stimuli further augments this secretion (Yu and Malenka, 2003). This occurs through $\mathrm{Ca}^{2+} /$ calmodulin-dependent protein kinase I and the transcription factor CREB, resulting in the development of dendrites (Wayman et al., 2006). Together, these data firmly establish the involvement of Wnt signaling in dendrite development. But the molecular mechanism of how Wnt signaling regulates specifically dendrite development remains unclear.

In this study, we perform functional analyses of Par1b/ MARK2 in hippocampal neurons and find that Par1b/MARK2 negatively regulates dendrite development. Wnt signaling induces translocation of Par1b/MARK2 from the cytosol to the membrane and blocks the negative effect of Parlb/MARK2 on dendrite development. In addition, we find that Par1b/MARK2 specifically phosphorylates MAP2, a microtubule-stabilizing protein that accumulates in dendrites, which may explain the dendrite-specific effect of Par1b/MARK2.

\section{Materials and Methods}

Plasmids. The full-length human Par1b/MARK2 cDNA (GenBank accession number AB271694) was cloned by PCR from a human embryonic kidney 293 (HEK293) cell cDNA library. Point mutations in Parlb/ MARK2 were introduced with a Site-directed Mutagenesis Kit (Stratagene, La Jolla, CA). The Src myristoylation signal sequence was added to Par1b/MARK2 using the PCR to generate Myr+Par1b/MARK2. MyrA2+Par1b/MARK2 differs from Myr+Par1b/MARK2 in the glycine on which myristoylation occurs is replaced with alanine. The full-length Dvll and Dvl-DEP/CT constructs were prepared in the previous study (Funato et al., 2006). The other deletion constructs of Dvll were generated by digestion with appropriate restriction enzymes or PCR. Amino acid residue numbers of the deletion constructs are as follows: $\triangle D I X 1$ (69-670), $\Delta$ DIX2 (244-670), PDZ (PSD-95/Dlg/ZO-1)/DEP (Dishevelled-Eg110-Pleckstrin) (69-499), PDZ/DEP2 (244-499) and DIX/PDZ (1-379).

Cell culture and transfection. L-Wnt3a cells were purchased from American Type Culture Collection (Manassas, VA). COS-7 and L cells were maintained in DMEM supplemented with $10 \%$ fetal bovine serum. NIH3T3 cells were maintained in DMEM with $10 \%$ calf serum. Cells were transfected with LipofectAMINE or LipofectAMINE 2000 (Invitrogen, Carlsbad, CA) according to the manufacturer's instructions. For the microtubule stabilization assay, cells were treated with $5 \mu \mathrm{M}$ nocodazole (Sigma, St. Louis, MO) for $1 \mathrm{~h}$ before fixation. Primary hippocampal cultures were prepared from embryonic day 18 rat embryos. Neurons $\left(10^{5}\right.$ cells $\left./ \mathrm{cm}^{2}\right)$ were seeded on poly-D-lysine and laminin-coated coverslips (BD Biosciences, San Jose, CA) in neurobasal medium and transfected after $2 \mathrm{~d}$ with LipofectAMINE 2000.

RNA interference. Duplex siRNAs for rat Par1b/MARK2 were purchased from Invitrogen. Nucleotide sequences were Parlb/MARK2-siRNA\#1, sense, 5' ${ }^{\prime}$ AAACCGAACACCGUUGAGAGACAGC-3' and antisense, 5' GCUGUCUCUCAACGGUGUUCGGUUU-3'; Par1b/MARK2-siRNA\#2, sense, 5'-AAUAAACUUCUGGUGACAGUACUGC-3' and antisense, 5' GCAGUACUGUCACCAGAAGUUUAUU- $3^{\prime}$ and negative control, sense, $5^{\prime}$-AAAUGGCGUCGCGUUCCUUUAGAGG-3' and antisense, $5^{\prime}$-CCUCUAAAGGAACGCGACGCCAUUU-3'.

Subcellular fractionation. Subcellular fractionation was performed as described by Behrmann et al (2004). In brief, cells were washed once with ice-cold PBS, collected with a cell scraper on ice and suspended in hypotonic buffer $[10 \mathrm{~mm}$ Tris- $\mathrm{HCl}, \mathrm{pH} 7.5,10 \mathrm{~mm} \mathrm{NaCl}, 3 \mathrm{~mm} \mathrm{MgCl}, 1 \mathrm{~mm}$ EDTA, 1 mM DTT, $0.1 \mathrm{~mm}$ phenylmethylsulfonyl fluoride (PMSF), 0.5 mu sodium orthovanadate, $10 \mu \mathrm{g} / \mathrm{ml}$ leupeptin, $10 \mu \mathrm{g} / \mathrm{ml}$ aprotinin]. Cells were incubated on ice for $15 \mathrm{~min}$ and then homogenized. The homogenate was centrifuged at $500 \mathrm{rpm}$ for $15 \mathrm{~min}$ at $4^{\circ} \mathrm{C}$ to remove nuclei. The supernatant was centrifuged again at $12,500 \times g$ at $4^{\circ} \mathrm{C}$. The resulting supernatant and pellet were used as the cytosol and the membrane fraction, respectively.

Cell lysis, immunoprecipitation, and Western blot analyses. Cells were harvested with lysis buffer (50 mM Tris- $\mathrm{HCl}, \mathrm{pH} 7.5,150 \mathrm{~mm} \mathrm{NaCl}, 5 \mathrm{~mm}$ EDTA, $0.5 \%$ Triton X-100, $0.1 \mathrm{~mm}$ PMSF, $0.5 \mathrm{~mm}$ sodium orthovanadate, $10 \mu \mathrm{g} / \mathrm{ml}$ leupeptin, $10 \mu \mathrm{g} / \mathrm{ml}$ aprotinin). Lysate were centrifuged at $15,000 \mathrm{rpm}$ at $4^{\circ} \mathrm{C}$, and the supernatant was incubated with appropriate antibodies and protein G-Sepharose (Pierce, Rockford, Il) at $4^{\circ} \mathrm{C}$ for $2 \mathrm{~h}$. Immunoprecipitates were washed four times with lysis buffer, separated by SDS-PAGE and transferred to a polyvinylidene difluoride membrane. Membranes were blocked with $10 \%$ fat-free dry milk in PBS with $0.05 \%$ Tween 20, incubated with primary antibodies and then incubated with alkaline phosphatase-conjugated anti-mouse IgG or anti-rabbit IgG (Promega, Madison, WI) for detection. Quantification by optical density was done with NIH Image software. Each experiment was repeated at least three times.

Immunofluorescence microscopy. Cells were washed with PBS and fixed with $3.7 \%$ formaldehyde in PBS for 15 min. Cells were then permeabilized with $0.2 \%$ Triton X-100 in PBS for 5 min. For tubulin staining, cells were fixed with methanol at on ice for $3 \mathrm{~min}$ and rehydrated three times for 5 min with PBS. After blocking with 2\% BSA in PBS for 10 min, cells were incubated for $1 \mathrm{~h}$ with the primary antibody diluted in blocking buffer. After three 5 -min washes with PBS, cells were incubated for 30 min with appropriate secondary antibodies diluted in blocking buffer. Washed with PBS, coverslips were mounted and immunofluorescence was observed with a confocal scanning laser microscope (Radiance 2000; Bio-Rad, Hercules, CA). Fluorescence images were processed with Adobe (San Jose, CA) Photoshop software.

Antibodies. The following antibodies were used: monoclonal anti- $\beta$ tubulin, monoclonal anti-Flag and monoclonal anti-MAP2 antibodies (Sigma); monoclonal anti-Taul antibody (clone PC16C that preferentially recognizes dephosphorylated Tau) (Millipore, Billerica, MA); monoclonal anti-human transferrin receptor antibody (Zymed, South San Francisco, CA); rabbit polyclonal anti-c-Myc antibody (Santa Cruz Biotechnology, Santa Cruz, CA); Alexa Fluor 488 goat anti-rabbit IgG and anti-mouse IgG, Alexa Fluor 546 goat anti-rabbit IgG and antimouse IgG and Alexa Fluor 633 goat anti-rabbit IgG (Invitrogen). AntiPar1b/MARK2 antibody (Suzuki et al., 2004) and anti-Dvl antibody (Funato et al., 2006) were prepared as parts of previous studies.

Protein purification and in vitro kinase assay. Recombinant Parlb/ MARK2 and recombinant Tau expressed in Escherichia coli (BL21) with an N-terminal His-tag was purified with Ni-NTA beads (Qiagen, Hilden, Germany). Purification of dephosphorylated Tau and MAP2 from porcine brains was performed as described previously (Itoh and Hotani, 1994). In brief, crude microtubules, which also contain several MAPs, were obtained by ultracentrifugation. Microtubules were dephosphorylated with calf-intestine alkaline phosphatase and then boiled for $5 \mathrm{~min}$ in the presence of $0.8 \mathrm{M} \mathrm{NaCl}$ and $50 \mathrm{~mm} \beta$-mercaptoethanol. The resulting dephosphorylated Tau and MAP2 were separated by gel filtration with Superdex 200 (GE Healthcare, Piscataway, NJ). Protein concentrations were determined by the Lowry method with bovine serum albumin as the standard. Kinase activities were assayed in $50 \mathrm{~mm}$ Tris- $\mathrm{HCl}, \mathrm{pH} 7.5,5 \mathrm{~mm}$ $\mathrm{MgCl}_{2}, 2 \mathrm{~mm}$ EGTA, $0.5 \mathrm{~mm}$ PMSF, and $0.5 \mathrm{~mm} \mathrm{DTT}$, at $30^{\circ} \mathrm{C}$ in the presence of $\left[\gamma^{-32} \mathrm{P}\right]$ ATP. Hymenialdisine (HD) (Biomol, Plymouth Meeting, PA) was added to a final concentration of $50 \mu \mathrm{M}$.

In vivo phosphorylation of MAP2. Examination of phosphorylation status of MAP2 was performed as described previously (Takenaka et al., 2007) with a minor modification. In brief, NIH3T3 cells were transfected with indicated constructs. After $48 \mathrm{~h}$ transfection, cells were washed twice with phosphate-free medium and incubated with $\left[{ }^{32} \mathrm{P}\right]$ orthophosphate at final concentration of $1 \mathrm{mCi} / \mathrm{ml}$ in phosphate-free medium for $6 \mathrm{~h}$, and then harvested with lysis buffer. The lysates were immunoprecipitated 
with anti-FLAG antibody, followed by Western blot analysis and autoradiography.

Statistics. All results are shown as mean \pm SEM. Significance was determined by twotailed Student's $t$ test.

\section{Results}

Ectopic expression of Par1b/MARK2 specifically inhibits dendrite development

To investigate the function of Parlb/ MARK2 after axon specification, we transiently expressed Flag-tagged Par1b/ MARK2 (Flag-Par1b/MARK2) in rat hippocampal neurons cultured for $2 \mathrm{~d}$, at which point polarity (axon specification) is known to have been established (Dotti et al., 1988). At $48 \mathrm{~h}$ after transfection, the control green fluorescence protein (GFP)expressing neurons showed normal neuronal morphology, consisting of a single long neurite (probably an axon) and several short neurites (probably dendrites). In contrast, neurons expressing wild-type (WT) Par1b/MARK2 had an abnormal morphology, with one long neurite and a few very short neurites (Fig. 1A). The morphology of neurons expressing the kinase-negative mutant $(\mathrm{KN})$ of Parlb/ MARK2 did not differ significantly from that of the control (GFP-expressing) neurons (Fig. $1 A$ ). To investigate the morphologic change in more detail, we performed staining analyses with Tau and MAP2, which are localized primarily in axon and dendrites, respectively, in cultured neurons after establishment of polarity (Kosik and Finch, 1987). We found that the single long neurite in Par1b/MARK2 WT expressing neuron was an axon $($ Fig. $1 B$ ). We then examined length and branching in axon or dendrites. The axon length of Flag-Par1b/MARK2 WT expressing neuron was a little longer than that of control GFP-expressing neuron (data not shown), as reported previously (Uboha et al., 2007). However, there was a clear difference in the morphology of dendrites. The control GFP-expressing neurons had a total dendrite length of 706.26 $\pm 32.23 \mu \mathrm{m}$, whereas Par1b/MARK2 WT expressing neurons had a length of $297.74 \pm 21.15$ $\mu \mathrm{m}(p<0.0001)$ (Fig. 1C). Expression of Par1b/MARK2 WT also decreased the number of dendrite branches, as quantified by the total dendrite branching tip number (TDBTN; 6.24 $\pm 0.40, p<0.0001$ ) compared with the GFP-expressing neurons $(16.01 \pm 0.70)($ Fig. $1 D)$. In contrast, expression of Par1b/MARK2 KN did not significantly affect the length or branching of dendrites. These results suggest that Par1b/MARK2 negatively regulates dendrite development, but not of axon, in a kinase activity dependent manner.

Expressed Flag-Par1b/MARK2 WT was observed in axons, dendrites, and cell body (Fig. $1 A, B$ ). Furthermore, we investigated lo-

A

B

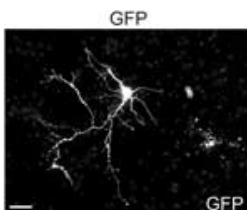

Flag-Par1b/MARK2 WT
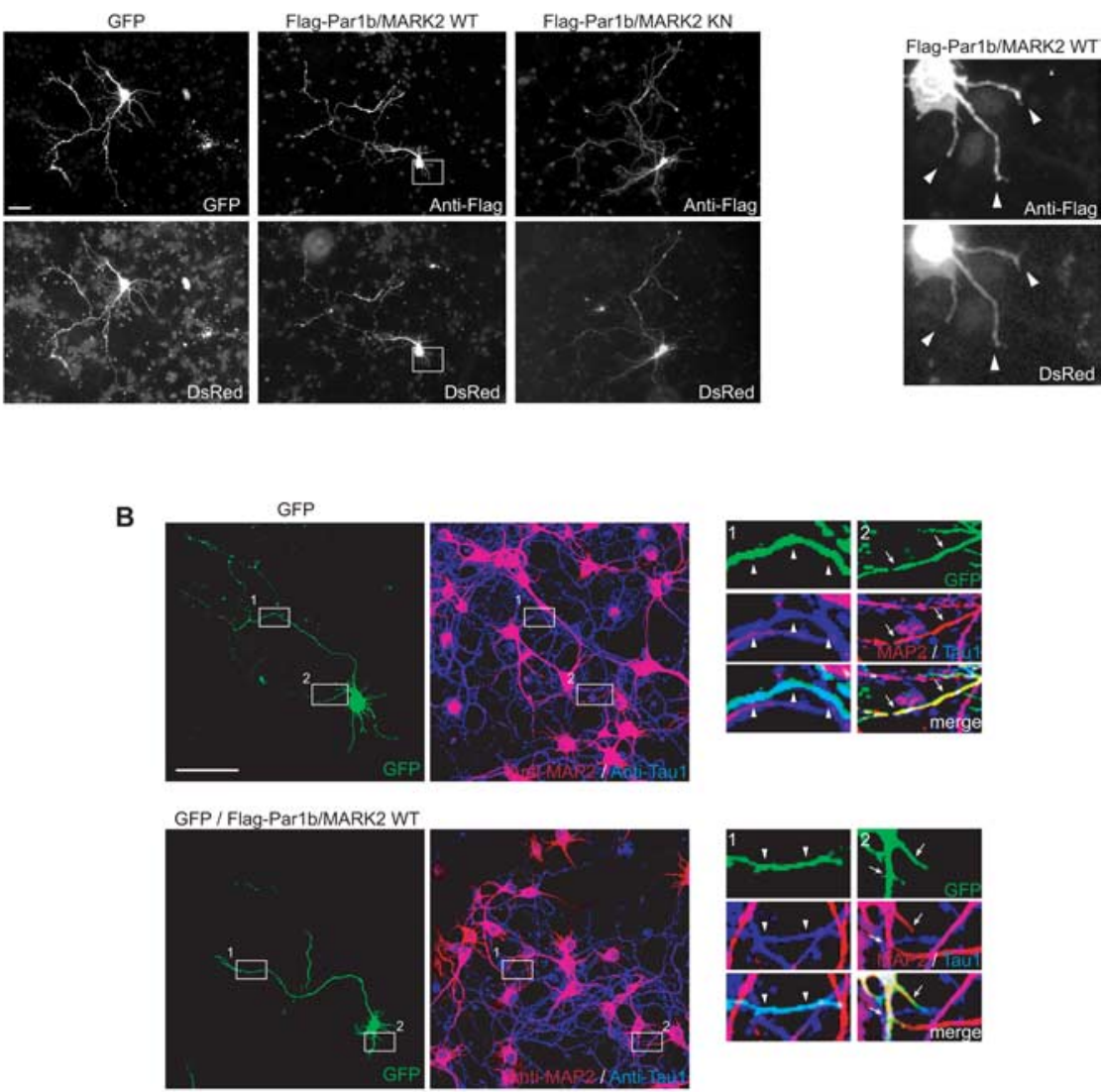

C

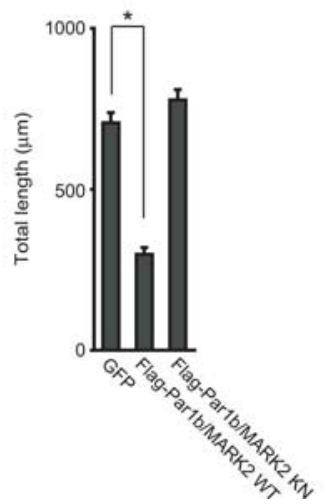

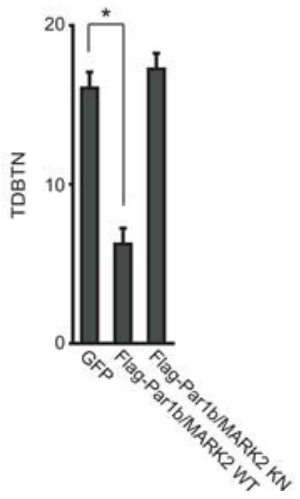

Figure 1. Expression of Par1b/MARK2 inhibits development of dendrites of rat hippocampal neurons. $\boldsymbol{A}$, Rat hippocampal neurons cultured for $2 \mathrm{~d}$ were transfected with DsRed and GFP, Flag-Par1b/MARK2 WT, or KN, and then subjected to immunofluorescence microscopy. Cells were visualized by direct observation of DsRed fluorescence in addition to GFP fluorescence or staining with anti-Flag antibody. The boxed areas are magnified and shown at the right side. Arrowheads indicate the tip of each neurites. Scale bar, $50 \mu \mathrm{m}$. B, Neurons expressing only GFP or GFP with Flag-Par1b/MARK2 WT were visualized by GFP fluorescence and staining with anti-MAP2 (red) and anti-Tau1 (blue) antibodies. The boxed areas are magnified and shown at the right side. Arrowheads and arrows indicate neurites that are positive for Tau and MAP2 staining, respectively. Scale bar, $100 \mu \mathrm{m}$. C, D, Quantification of a total dendrite length $(\boldsymbol{C})$ and TDBTN (total dendrite branching tip number, $\boldsymbol{D}$ ) of neurons transfected with the indicated constructs. All data are mean \pm SEM $(n>30) .{ }^{*} p<0.0001$

calization of endogenous Par1b/MARK2. The anti-Par1b/MARK2 antibody, which was used for immunofluorescence analyses in MDCK epithelial cells, clearly recognized endogenous proteins in neurons in Western-blotting analysis (supplemental Fig. $1 \mathrm{~A}$, available at www.jneurosci.org as supplemental material). Using this antibody, we performed immunofluorescence analysis in hippocampal neurons, and found that endogenous Par1b/MARK2 localized in a similar manner as expressed Flag-Par1b/MARK2 (supplemental Fig. $1 B$, available at www.jneurosci.org as supplemental material). These 
A

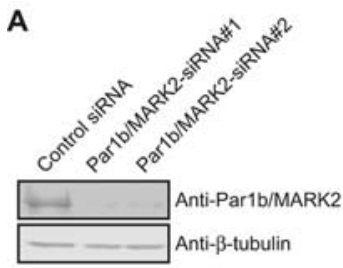

B

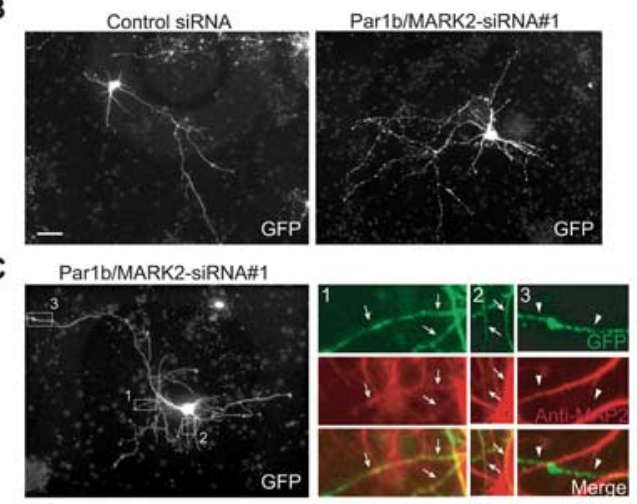

D

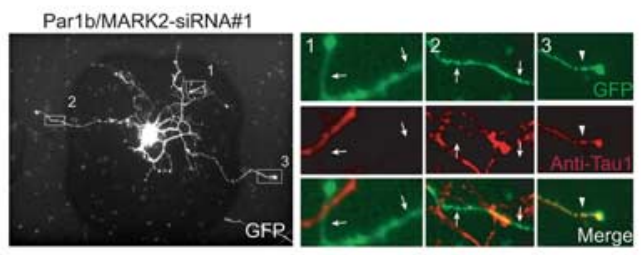

E

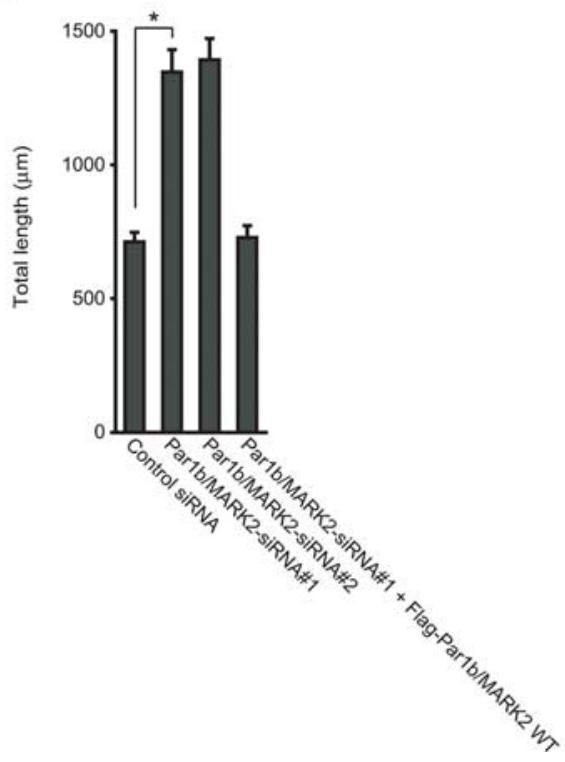

$\mathbf{F}$

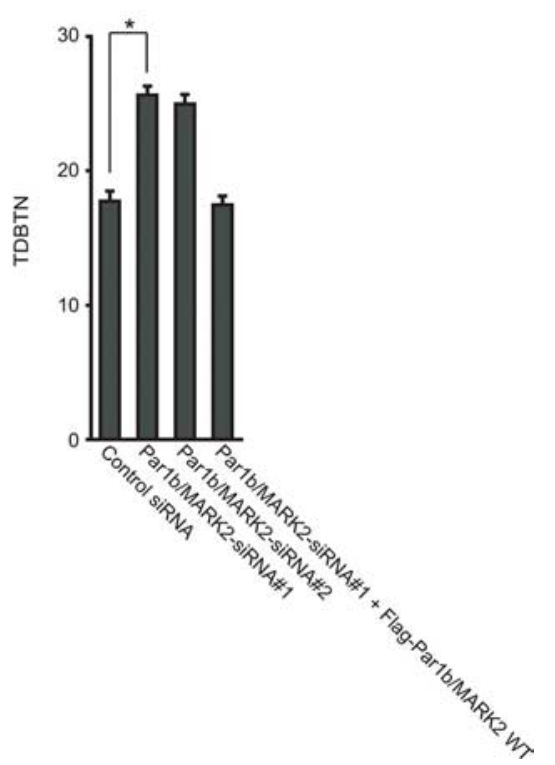

Figure 2. RNA interference-mediated knockdown of Par1b/MARK2 induces dendrite development. $\boldsymbol{A}, 3 \mathrm{Y} 1$ cells were transfected with the indicated siRNAs, and lysates were subjected to immunoblotting with anti-Par1b/MARK2 antibody and anti- $\beta$ tubulin antibody. $\boldsymbol{B}$, Hippocampal neurons cultured for $2 \mathrm{~d}$ were transfected with the indicated siRNAs and GFP for visualization of cells. $\boldsymbol{C}, \boldsymbol{D}$, Neurons transfected with Par1b/MARK2-siRNA\#1 were visualized by cotransfected GFP and immunostaining with anti-MAP2 ( $\boldsymbol{C}$ or anti-Tau1 (D) antibodies, respectively. The boxed areas are magnified and shown at the right side (GFP, green; MAP2 or Tau, red). Merged images are also indicated. Arrows indicate neurites with positive for MAP2 staining ( $C$ ) and negative for Tau staining $(\boldsymbol{D})$. Arrowheads indicate neurites with negative for staining of MAP2 $(\boldsymbol{C})$ and positive for staining of Tau (D). Scale bars: $\boldsymbol{B}-\boldsymbol{D}, 50 \mu \mathrm{m} . \boldsymbol{E}, \boldsymbol{F}$, Quantification of a total dendrite length $(\boldsymbol{E})$ and TDBTN $(\boldsymbol{F})$ of neurons transfected with the indicated siRNAs. Data are mean \pm SEM $(n>30) .{ }^{*} p<0.0001$.

results implicate the possibility that Par1b/MARK2 can function in both axons and dendrites.

\section{Dendrite development in response to RNA interference- mediated knockdown of Par1b/MARK2}

To confirm the involvement of Par1b/MARK2 in dendrite development, we performed a loss-of-function analysis of endogenous Parlb/MARK2. We used small-interfering (si) RNAs to reduce expression of Par1b/MARK2. We found that two different siRNAs, Par1b/MARK2-siRNA\#1 and Par1b/MARK2-siRNA\#2, efficiently suppressed expression of endogenous Par1b/MARK2 in

$3 Y 1$ rat embryonic fibroblasts (Fig. 2A). We next transfected rat hippocampal neurons with these siRNAs together with GFP as a marker for visualization of neuron morphology. Neurons transfected with each siRNA had longer and more branched neurites than did control siRNA-transfected neurons (Fig. 2B). Immunostaining of MAP2 and Tau revealed that the long neurites that were formed after Par1b/MARK2-siRNA transfection were dendrites (Fig. 2C,D). When we quantified the total length and branching tip numbers of the dendrites, we found that the length was longer and the numbers were significantly higher in Parlb/ MARK2 knock down cells than in control cells (Fig. 2E,F).

To further confirm that these effects were the consequence of specific suppression of endogenous Par1b/MARK2, we cotransfected cells with a plasmid expressing human Par1b/MARK2 and Par1b/ MARK2-siRNA\#1. The Parlb/MARK2siRNA\#1 was designed against rat Parlb/ MARK2, and there are several mismatches between the nucleotide sequences of Par1b/MARK2-siRNA\#1 and the corresponding region of human Parlb/ MARK2. We confirmed that expressed human Par1b/MARK2 was resistant to the effect of Par1b/MARK2-siRNA\#1 (supplemental Fig. 2, available at www. jneurosci.org as supplemental material). The coexpression studies in neurons revealed that exogenously expressed Par1b/ MARK2 rescued the phenotype caused by Parlb/MARK2-siRNA (Fig. 2E,F). Together with the results of our overexpression analysis (Fig. 1), it is clear that Par1b/ MARK2 negatively regulates dendrite development.

\section{Par1b/MARK2 functions \\ antagonistically against Dvl in \\ dendrite development}

Dishevelled (Dvl) is an essential component of Wnt signaling (Wallingford and Habas, 2005; Malbon and Wang, 2006), which regulates dendrite development (Rosso et al., 2005). It has been reported that Dvl interacts with Par1 in Drosophila (Sun et al., 2001). Thus, we examined the possible relation between Par1b/MARK2 and Dvl during dendrite development. When we ectopically expressed Dvl in hippocampal neurons, they had longer and more branched dendrites than did the control neurons (Fig. 3A), as reported previously (Rosso et al., 2005). In contrast, coexpression of Par1b/MARK2 WT with Dvl blocked the effect of Dvl overexpression on elongation and branching of dendrites (Fig. 3 B, C). Par1b/MARK2 KN had no significant effect. These results suggest that Par1b/MARK2 has an antagonistic effect on Dvl in dendrite development (Fig. $3 B, C$ ). 


\section{Recruitment of Par1b/MARK2 to the} membrane fraction by Dvl expression or Wnt stimulation

We then addressed the molecular mechanism of the antagonistic action of Parlb/ MARK2 on Dvl. Par1b/MARK2 phosphorylates MAPs (Illenberger et al., 1996; Drewes et al., 1997), and our experiments using the KN mutant of Par1b/MARK2 implicated that the kinase activity of Par1b/MARK2 is important in dendrite development. Therefore, we first examined whether Dvl inhibited the kinase activity of Par1b/MARK2. Although we performed in vitro kinase assays with myelin basic protein as the substrate of anti-Flag immunoprecipitates of lysates of COS-7 cells expressing Flag-Par1b/MARK2, we did not observe any significant change in the kinase activity after coexpression of Dvl (supplemental Fig. 3, available at www.jneurosci.org as supplemental material). However, the amount of Flag-Par1b/ MARK2 in the membrane fraction was increased in response to coexpression of Dvl (Fig. $4 A$ ), and in the presence of Dvl, the signal of Flag-Par1b/MARK2 in the membrane fraction showed a significant shift toward higher molecular weight (Fig. 4A). This mobility shift is attributable to phosphorylation because the shift was absent after alkaline phosphatase treatment (supplemental Fig. $4 A$, available at www.jneurosci.org as supplemental material). LKB1, MARK kinase (MARKK) and aPKC are reported to phosphorylate Par1b/MARK2. LKB1 and MARKK phosphorylate Thr208 in the activation loop of Parlb/ MARK2 (Timm et al., 2003; Lizcano et al., 2004), and aPKC phosphorylates Thr-595 in the shaft region of Parlb/MARK2 (Hurov et al., 2004; Kusakabe and Nishida, 2004; Suzuki et al., 2004). We therefore examined whether these residues were involved in the mobility shift or translocation of Par1b/MARK2 in the presence of Dvl, but we did not observe so significant differences between WT and the Ala-substituted mutants for the phosphorylation sites (supplemental Fig. 4B, $C$, available at www.jneurosci.org as supplemental material). We examined whether phosphorylation of endogenous Parlb/ MARK2 occurred in response to Wnt stimulation, not only to Dvl expression. However, we did not observe any shift in the mobility of Par1b/MARK2 in cells treated with Wnt3aconditioned medium (CM) (data not shown) and, thus, we did not perform more detailed analyses of the phosphorylation.

We next examined whether Par1b/MARK2 was recruited to the membrane fraction in response to Wnt stimulation in neurons. Rat hippocampal neurons were treated with Wnt3a CM, and cell lysates were separated into cytosol and membrane fractions. The amount of Par1b/MARK2 in the membrane fraction increased in neurons treated with Wnt3a CM (Fig. 4B,C). Dvl was also recruited to the membrane fraction in response to Wnt3a CM (Fig. 4B,D). These results strongly suggest that Dvl recruits Par1b/MARK2 to the membrane fraction, and that Par1b/MARK2 was regulated by Wnts.

Because Wnt treatment induces dendrite development, we hypothesized that cytosolic Par1b/MARK2 negatively regulates dendrite development. To address this issue, we constructed Myr+Par1b/MARK2, which has a myristoylation sequence to force Par1b/MARK2 to the membrane, and MyrA2+Par1b/ MARK2, which acts as an inactive control because the myristoylated glycine was converted to alanine (Fig. $4 E$ ). We confirmed that Myr+Par1b/MARK2 was present exclusively in the membrane fraction, whereas MyrA2+Par1b/MARK2 and Par1b/ MARK2 WT were present in both membrane and cytosolic fractions (Fig. $4 F$ ). We transfected hippocampal neurons with these Par1b/MARK2 mutants (Fig. 4G). As expected, the Myr+Parlb/ MARK2-expressing neurons had a total dendrite length of $793.24 \pm 44.32 \mu \mathrm{m}$, which is similar to that of the control GFPexpressing neurons, whereas the MyrA2+Par1b/MARK2expressing neurons had a total dendrite length of $415.31 \pm 32.77$ $\mu \mathrm{m}$ (Fig. $4 H$ ). Expression of MyrA2+Par1b/MARK2 also decreased TDBTN, as did Par1b/MARK2 WT, whereas expression of Myr+Par1b/MARK2 had no effect on TDBTN (Fig. 4I). These 
A

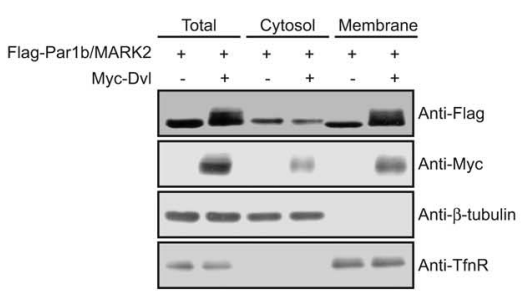

C

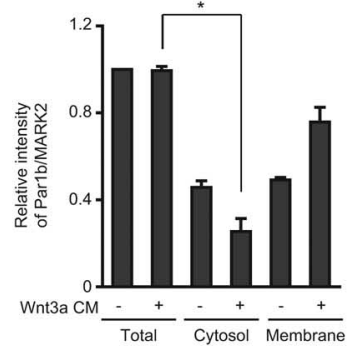

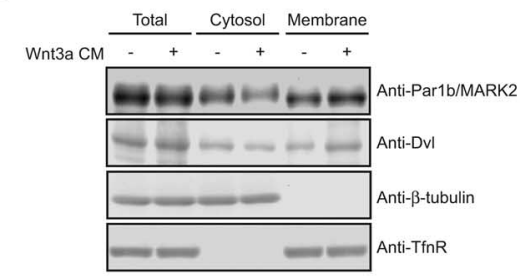

D

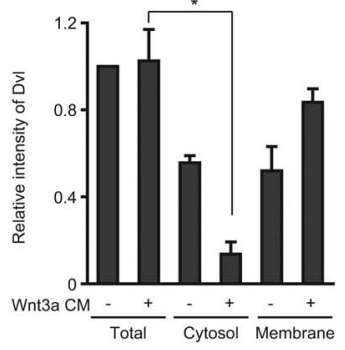

F

E

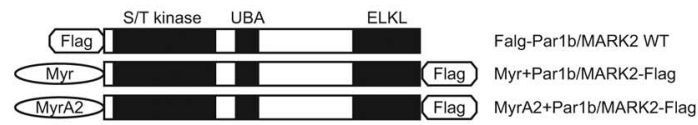

G
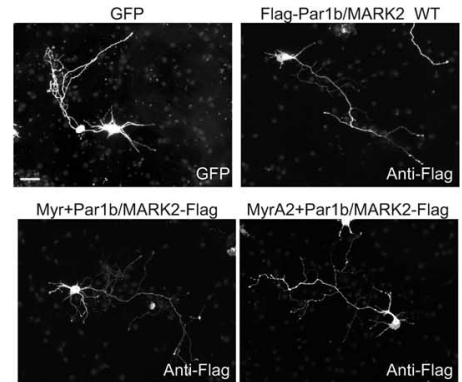

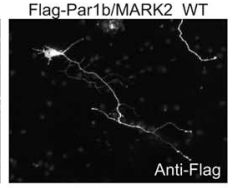

3.

Anti-Flag
H

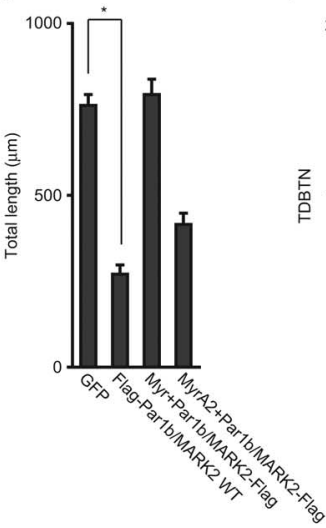

Figure 4. Recruitment of Par1b/MARK2 to the membrane fraction by Dvl expression or Wnt stimulation. $\boldsymbol{A}$, Lysates of COS-7 cells expressing Flag-Par1b/MARK2 with or without Myc-Dvl were separated into the cytosolic and membrane fractions. Each fraction was analyzed by immunoblotting with indicated antibodies. Anti- $\beta$-tubulin antibody and anti-transferrin receptor (TfnR) antibody were used as markers of cytosolic and membrane fractions, respectively. $\boldsymbol{B}$, Lysates of hippocampal neurons treated with Wnt3a CM (indicated as +) or control CM (indicated as -) were separated and subjected to immunoblotting analyses with the indicated antibodies. C, D, Quantification of the relative intensity of each band on immunoblots probed with anti-Par1b/MARK2 $(\boldsymbol{C})$ and anti-Dvl $(\boldsymbol{D})$ antibodies. All data are mean \pm SEM $(n=3) .{ }^{*} p<0.01$. $\boldsymbol{E}$, Schematic diagrams of Par1b/MARK2 mutants, Myr + Par1b/MARK2 and MyrA2 + Par1b/MARK2. $\boldsymbol{F}$, Lysates of COS-7 cells expressing Par1b/MARK2 mutants were separated into cytosolic and membrane fractions and examined by immunoblotting. $\mathbf{G}$, Representative images of hippocampal neurons expressing Par1b/MARK2 mutants. Scale bar, $50 \mu \mathrm{m}$. $\boldsymbol{H}, \boldsymbol{I}$, Quantification of total dendrite length $(\boldsymbol{H})$ and TDBTN $(I)$ of neurons transfected with the indicated constructs. All data are mean \pm SEM $(n>35)$. ${ }^{*} p<0.0001$.

results support the above mentioned hypothesis and suggest that regulation of intracellular localization of Parlb/MARK2 is important for dendrite development induced by Wnt.

\section{Microtubule-stabilizing activity of Dvl correlates with the interaction with Par1b/MARK2}

Because neurite extension occurs through reorganization of microtubules and because Dvl has been shown to be involved in microtubule stabilization (Krylova et al., 2000), we next exam-

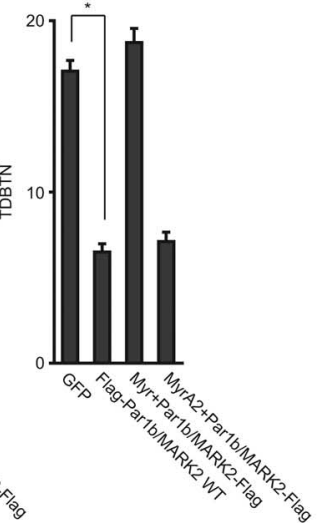

ined the relationship of Dvl and Parlb/ MARK2 in microtubule stabilization. We expressed Dvl in NIH3T3 cells and examined the ability of Dvl to stabilize microtubules (Fig. 5A). Consistent with a previous report (Krylova et al., 2000), expression of Dvl prevented destabilization of microtubules by nocodazole. We then examined the microtubule stabilizing activity of a series of Dvl-deletion constructs (Fig. $5 B, C)$. Among the Dvl constructs, Dvl$\triangle$ DIX1, Dvl-PDZ/DEP1, and Dvl-DIX/ PDZ stabilized microtubules similar to fulllength Dvl, whereas other deletion mutants did not induce stabilization. These results suggest that the PDZ domain and surrounding sequences are necessary for microtubule stabilization by Dvl, which is in agreement with a previous report (Krylova et al., 2000).

We next examined the interaction between Dvl and Par1b/MARK2. The direct interaction between recombinant $\mathrm{Dvl}$ and Par1b/MARK2 was first analyzed. Pulldown assays with purified GST-Dvl-His and His-Par1b/MARK2 indicated their direct interaction (supplemental Fig. 5A, available at www.jneurosci.org as supplemental material). Furthermore, the interaction between Dvl and Par1b/MARK2 in vivo was also observed (supplemental Fig. $5 B$, available at www.jneurosci.org as supplemental material). Then, we investigated the region of Dvl responsible for the interaction with Par1b/MARK2. Coexpression and immunoprecipitation analyses revealed that Dvl- $\Delta$ DIX1 and Dvl-PDZ/ DEP1 associated with Par1b/MARK2. Dvl-DIX/PDZ also associated with Parlb/ MARK2, although this interaction appeared to be weaker than that of the fulllength construct (Fig. 5B,D). Therefore, the binding ability of Dvl with Parlb/ MARK2 correlates well with microtubule stabilizing activity.

On the basis of these results, it was thought that regulation of microtubule stability by Dvl and Par1b/MARK2 should be important for dendrite development. We directly examined the effect of Parlb/ MARK2 on microtubule stabilization induced by Dvl. Coexpression of Parlb/ MARK2 WT with Dvl clearly blocked the microtubule stabilization induced by Dvl. In contrast, coexpression of Parlb/ MARK2 KN had no significant effect on Dvl-induced microtubule stabilization (Fig. 5E,F). Moreover, coexpression of Myr+Parlb/MARK2 also had no significant effect on Dvlinduced microtubule stabilization, whereas MyrA2+Parlb/ MARK2 did (Fig. 5F). These data suggest that intracellular localization of Par1b/MARK2 and its kinase activity are also important for microtubule stability. Then, we examined whether Dvl could induce microtubule stabilization in neurons. As shown in Figure 5G, Dvl induced microtubule stabilization also in neu- 
rons as well as in fibroblasts. Neurons expressing Myc-Dvl had stabilized microtubules after nocodazole treatment (42.32 \pm $3.34 \%$ ), whereas control neurons did not $(13.32 \pm 1.59 \%)$. These results are consistent with the previous report that showed microtubule stabilization by Dvl in cerebellar neurons (Ciani et al., 2004).

\section{Par1b/MARK2 efficiently phosphorylates MAP2}

How does Par1b/MARK2 selectively suppress dendrite development? It has been reported that Par1b/MARK2 phosphorylates MAPs, such as Tau and MAP2, to destabilize microtubules. Both Tau and MAP2 have two KXGS motifs (diagrammed in Fig. 6A) that are phosphorylated by Par1b/MARK2 in vitro. If Par1b/ MARK2 phosphorylates MAP2 more efficiently than Tau, the selective effect of Par1b/MARK2 on dendrite development can be explained, because MAP2 is localized primarily in dendrites (Kosik and Finch, 1987). To examine the validity of this hypothesis, we performed in vitro kinase assays of Par1b/MARK2 using Tau and MAP2 as substrates. We purified Tau and MAP2 from porcine brains and recombinant human Tau (rTau) from $E$. coli. Because brain Tau and MAP2 are significantly phosphorylated, we treated them with alkaline phosphatase during purification procedure (for details, see Materials and Methods). We confirmed that these proteins were efficiently dephosphorylated by the treatment and that the amount of phosphates remained in Tau was smaller than that in MAP2 (data not shown). In vitro kinase assays showed that MAP2 was strongly phosphorylated by recombinant Parlb/ MARK2 (Fig. 6B), whereas phosphorylation of brain Tau or rTau was much less prominent. This phosphorylation was inhibited by $\mathrm{HD}$, an inhibitor of Par1b/MARK2 kinase (Fig. 6B). Moreover, time course analysis confirmed that phosphorylation of brain Tau or rTau was much slower than that of MAP2 (Fig. 6C). These results suggest that substrate preference may underlie the specific effect of Par1b/MARK2 on dendrites.

Finally, we examined whether Dvl suppresses phosphorylation of MAP2 in vivo.

We transfected NIH3T3 cells with Flag-MAP2c alone or together with Myc-Dvl. Transfected cells were incubated with $\left[{ }^{32} \mathrm{P}\right]$ orthophosphate and their lysates were subjected to immunoprecipitation with anti-Flag antibody. The phosphorylation status of MAP2c was examined by autoradiography. We found an $\sim 40 \%$ decrease in the radioactivity of MAP2c coexpressed with Dvl, compared with MAP2c alone (Fig. 6D).
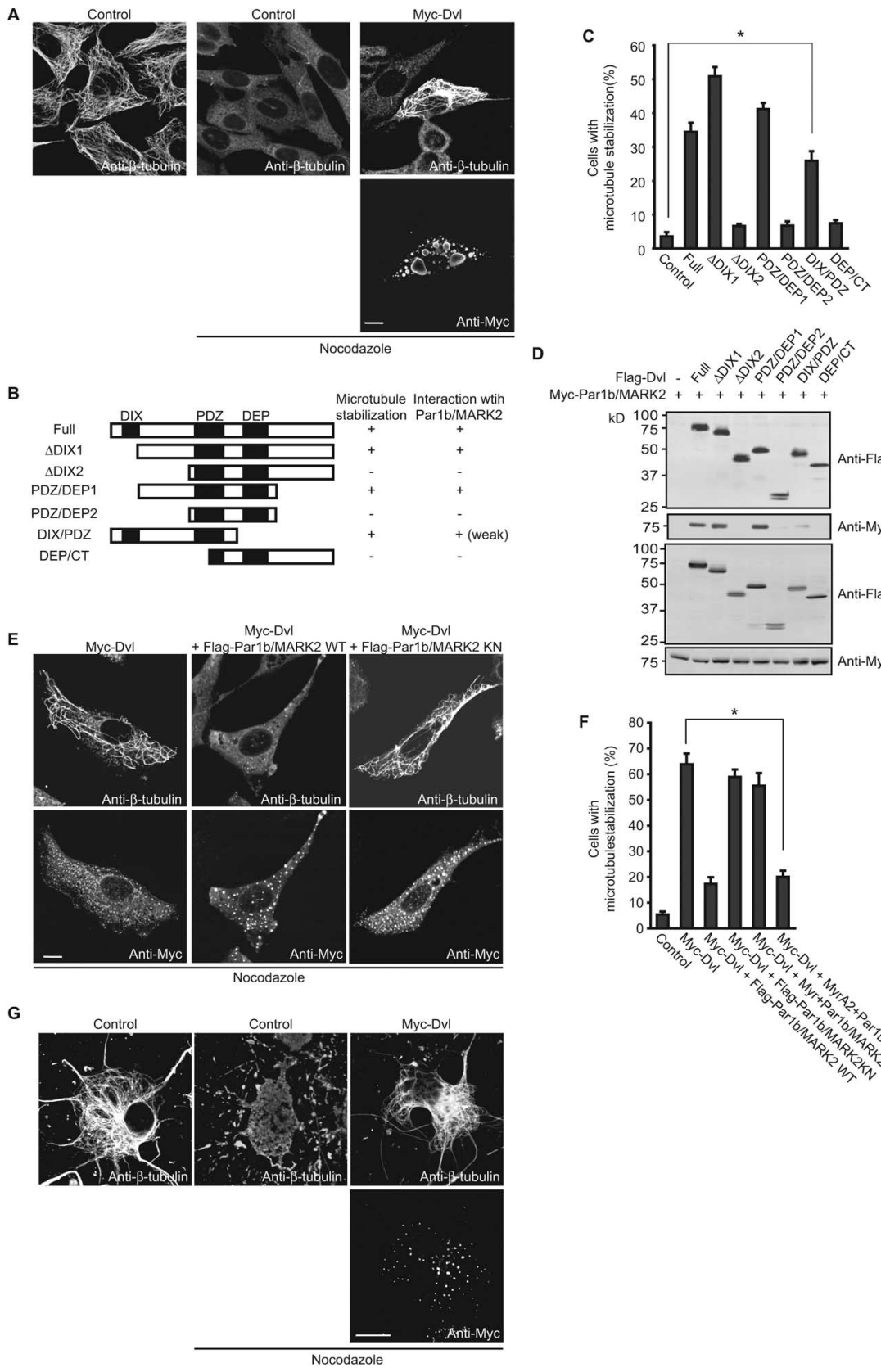

Figure 5. Par1b/MARK2 inhibits microtubule stabilization induced by Dvl. $\boldsymbol{A}, \mathrm{NIH} 3 \mathrm{~T} 3$ cells were transfected with Myc-Dvl. At $24 \mathrm{~h}$ after transfection, cells were treated with $5 \mu \mathrm{m}$ nocodazole for $1 \mathrm{~h}$ and stained with anti-Myc antibody and anti- $\beta$-tubulin antibody. Representative images of $\beta$-tubulin-stained nontransfected cells treated with or without nocodazole are also shown. Scale bar, $5 \mu \mathrm{m}$. $\boldsymbol{B}$, Schematic diagrams of deletion constructs of Dvl. Microtubule stabilizing activity and interactions with Par1b/MARK2 are summarized on the right side of each construct. + , Positive; - , negative. $C$, Quantification of microtubule stabilizing activity of Dvl constructs. Cells contained microtubules after nocodazole treatment were counted. All data are mean \pm SEM $(n=3)$. The asterisk indicates $p<0.01$. D, Myc-Par1b/MARK2 and Flag-tagged deletion constructs of Dvl were expressed in COS-7 cells and then immunoprecipitated with anti-Flag antibody. The precipitates were analyzed by immunoblotting. $\boldsymbol{E}$, NIH3T3 cells transfected with indicated plasmids were treated with nocodazole. Cells were stained as described in $(\boldsymbol{A})$. Scale bar, $5 \mu \mathrm{m}$. $\boldsymbol{F}$, Quantification of microtubule stabilization in cells expressing indicated constructs. All data are mean $\pm \operatorname{SEM}(n=3) .{ }^{*} p<0.01$. G. Microtubule stabilization in hippocampal neurons. Scale bar, $10 \mu \mathrm{m}$.

\section{Discussion}

Several groups have previously demonstrated the function of Par1b/MARK2 in axon development. Chen et al. (2006) and Zhang et al. (2007) introduced expression constructs and specific siRNAs for Par1b/MARK2 into neurons before establishment of axon/dendrite polarity, and found that it inhibits axon formation. However, Uboha et al. (2007) examined the effect of Par1b/ 


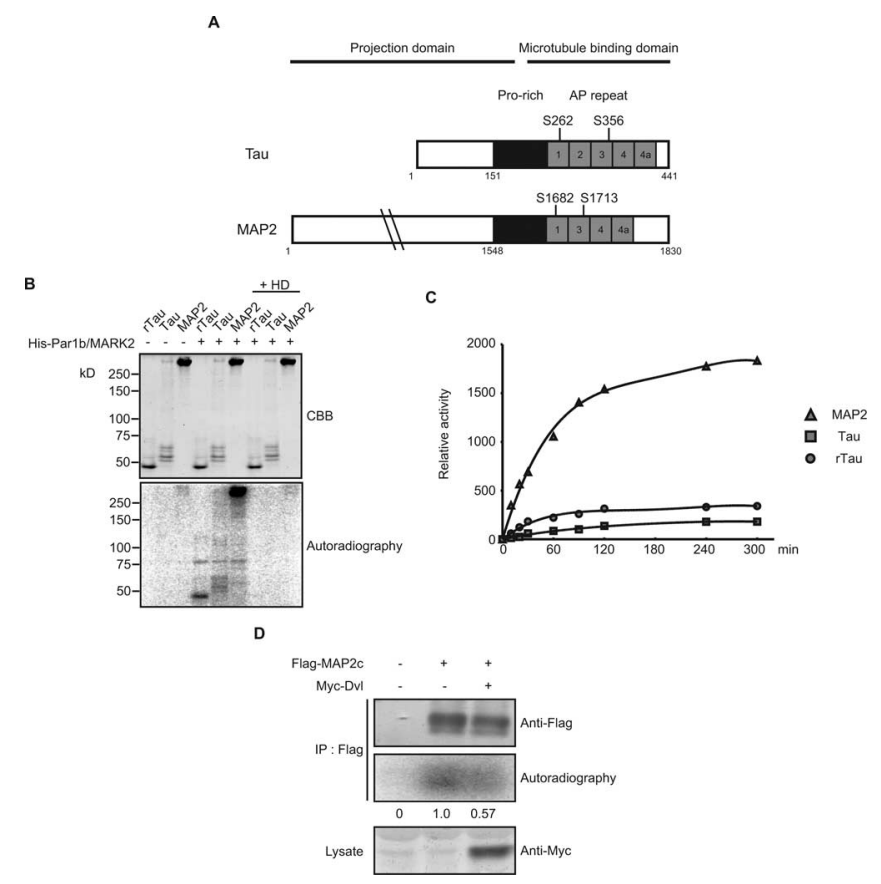

Figure 6. Substrate specificity of Par1b/MARK2 kinase activity. $\boldsymbol{A}$, Schematics of Tau (human isoform Tau40) and MAP2. Bar diagrams show the N-terminal projection domain and the C-terminal microtubule-binding domain, which include the proline-rich (pro-rich) region and the assembly-promoting (AP) repeat region. Residues known to be phosphorylated by Par1b/ MARK2 in vitro are indicated. $\boldsymbol{B}$, Purified porcine brain MAP2 and Tau (0.1 $\mu \mathrm{g}$ each), and recombinant Tau from $E$. coli ( $\mathrm{rTau}, 0.1 \mu \mathrm{g}$ ) were subjected to kinase assays by recombinant Par1b/MARK2 protein. HD was added to a final concentration of $50 \mu \mathrm{m}$. The reactions were stopped by the addition of SDS-PAGE sample buffer. The proteins were separated by SDS-PAGE and then subjected to autoradiography. $C$, Time course of phosphorylation of each MAP ( 2 pmol each). The triangle, square, and circle indicate the relative radioactivities of MAP2, Tau and rTau, respectively. D, Dvl inhibits phosphorylation of MAP2. Flag-MAP2c was expressed with or without Myc-Dvl in NIH3T3 cell. After $48 \mathrm{~h}$ of transfection, cells were labeled with [ ${ }^{32} \mathrm{P}$ ]orthophosphate. Lysates were immunoprecipitated with anti-Flag antibody and then precipitates were subjected to immunoblotting and autoradiography.

MARK2 in axon elongation after polarity establishment and discovered that Par1b/MARK2 augments the elongation of axons. Therefore, the importance of Par1b/MARK2 in axon development is complicated before/after polarity establishment and it will require more future studies to precisely understand the function and regulation of Par1b/MARK2 in axon development. In contrast, we examined here the importance of Par1b/MARK2 in dendrite development and showed that it negatively regulates dendrite development, which is antagonized by Wnt signaling. This is the first demonstration of the importance of Parlb/ MARK2 in dendrite development and clearly links Wnt signaling to dendrite development.

Axons and dendrites are not only different in their morphology and function but also in their molecular composition. It is widely accepted that Tau and MAP2 localize predominantly in axons and dendrites, respectively (Kosik and Finch, 1987). Studies of hippocampal neurons obtained from the double-knockout mice of the Tau or MAP2 gene with the MAP1B gene confirmed that the Tau and MAP2 genes are important in normal development of axons and dendrites, respectively (Takei et al., 2000; Teng et al., 2001). However, the regulatory mechanisms of Tau and MAP2 during development of axons and dendrites remain mostly unknown. In the present study, we have shown that Par1b/MARK2 preferentially phosphorylates MAP2. MARK was originally identified by biochemical purification from porcine brains as a kinase that phosphorylates MAP2, MAP4 and Tau (Illenberger et al., 1996). It was reported that recombinant Par1/ MARK phosphorylates the KXGS motif of Tau (Drewes et al., 1997). However, our quantitative and comparative analyses of purified dephosphorylated Tau and MAP2 clearly indicated a significant difference in the efficiency of phosphorylation between Tau and MAP2. We also tested the phosphorylation efficiency against recombinant human Tau and obtained similar results. Therefore, we concluded that Par1b/MARK2 preferentially phosphorylates MAP2 over Tau. We should mention that a certain amount of Tau actually exists in dendrites and is phosphorylated to some extent (Papasozomenos and Binder, 1987; Kanai and Hirokawa, 1995; Hirokawa et al., 1996). The existence of Tau in dendrites raises a possibility that Tau may also affect dendrite development. However, Tau is very abundant in axons and only a minor fraction of it exists in dendrites (Binder et al., 1985; Peng et al., 1986; Kosik and Finch, 1987; Kanai and Hirokawa, 1995; Hirokawa et al., 1996). In addition, the comparative studies on the Tau and MAP1B double-knock-out mice and the MAP1B single-knock-out mice clearly indicated the importance of Tau on axons without so significant effect on dendrites (Takei et al., 2000). These reports suggest that Tau should be essential for the elongation of axons and play only minor, if any, contribution to dendrite development after polarity establishment. Hence, the substrate preference we have shown here presumably contributes to the dendrite-specific effect of Par1B/MARK2. However, it is possible that Par1b/MARK2 is inactivated specifically in axons through unknown mechanism.

In addition, Rosso et al. (2005) reported the importance of Rac and c-Jun-N-terminal kinase (JNK) in dendrite development. They demonstrated that Dvl promoted dendrite development through activation of Rac and JNK. It is also reported that one splice variant Xenopus Par1 was involved in activation of JNK (Ossipova et al., 2005). However, the relation of Rac and JNK with Par1b/MARK2 also remains unclear. Further studies are needed to clarify the role of each of these molecules in dendrite development.

As for the antagonistic relationship between Par1b/MARK2 and Dvl, it was previously shown that overexpression of Parlb/ MARK2 attenuated Dvl signaling in epithelial cells (Elbert et al., 2006). In epithelial cells, Dvl decreased cell-cell adhesion through suppression of the E-cadherin function, which was antagonized by Par1b/MARK2. This report not only supports our finding in neurons, but also raises the possibility that Par1/MARK may inhibit dendrite development by regulating the functions of cadherin family proteins. Indeed, $\mathrm{N}$-cadherin and Flamingo/Celsr, a single-pass and seven-pass transmembrane cadherins, respectively, have been shown necessary for dendrite development and patterning (Gao et al., 2000; Yu et al., 2003). The possible functional relationship between these cadherin family proteins and Par1b/MARK2, if any, may be an interesting theme for future studies.

Par1b/MARK2-deficient mice are already reported. These mice are viable, displaying growth retardation and immune system dysfunction (Bessone et al., 1999; Hurov et al., 2001). Because we and other groups proposed the involvement of Parlb/ MARK2 in neuronal morphology, Par1b/MARK2-deficient mice would have defects in neuronal development, which may lead to embryonic lethality. Therefore, the fact that mice lacking Par1b/ MARK2 are not lethal may be interesting. However, it is known that Dvl1-deficient mice, of which hippocampal neurons in primary culture also show severe defects in dendrite development (Rosso et al., 2005), are also viable (Lijam et al., 1997). Therefore, 
such defects observed in in vitro culture may be overcome or some compensatory mechanism may be working in neurons in in vivo situation.

As described above, the localization of Par1b/MARK2 is regulated via phosphorylation of T595 by aPKC (Hurov et al., 2004; Kusakabe and Nishida, 2004; Suzuki et al., 2004). Previously, there appeared another report that one of the family kinases MARK3 is also phosphorylated by aPKC not only on T595 but also on several other residues, which collectively contribute to the regulation of Par1b/MARK2 localization (Goransson et al., 2006). However, there is a common feature in these reports that the localization of Par1b/MARK2 is regulated by phosphorylation from the membrane to the cytosol. In this point, it should be noted that our study has revealed a new mechanism of the localization regulation by the Wnt signaling. We observed significant recruitment of Par1b/MARK2 protein in the membrane in response to expression of Dvl (Fig. 4A). Furthermore, we found that Wnt stimulation induced the recruitment of cytosolic Par1b/ MARK2 to the membrane. It is thought that this is caused by the interaction of Par1b/MARK2 with Dvl because Dvl also translocates from the cytosol to the membrane and Dvl binds to Parlb/ MARK2. This suggests the interesting possibility that it is cytosolic Parlb/MARK2, not membranous Par1b/MARK2, that negatively affects dendrite development. To test this possibility, we generated a membrane-targeting construct of Par1b/MARK2 (Myr+Par1b/MARK2) and confirmed that the membranetargeted mutant had no effect on dendrite development. This result supports our hypothesis, but there is another possibility that the membrane-targeted mutant may not be accessible to its activating kinases, which leaves Par1b/MARK2 inactive. Additional studies are needed to clarify this problem. To our knowledge, three groups have reported that aPKC phosphorylates Par1/ MARK, causing it to move from the membrane to the cytosol (Hurov et al., 2004; Kusakabe and Nishida, 2004; Suzuki et al., 2004). One group claimed that the phosphorylation by aPKC also inhibits the kinase activity (Hurov et al., 2004), but the other two groups reported that there is no significant change in kinase activity (Kusakabe and Nishida, 2004; Suzuki et al., 2004). This remains a controversial issue, but such aPKC-mediated phosphorylation of Par1/MARK is generally thought to negatively regulate the function of Par1/MARK. Our study is the first to suggest the importance of cytosolic Par1/MARK.

MAP2 is a microtubule-binding and -stabilizing protein that exists mainly in the cytoplasm. This is consistent with the function of Par1/MARK in the cytosol. Together, these data suggest that the regulation of Par1/MARK localization by aPKC or some other mechanism is not a simple on-off switch but is critical for changing the access to its substrates in the cytosol or membrane.

\section{References}

Arimura N, Kaibuchi K (2005) Key regulators in neuronal polarity. Neuron 48:881-884.

Binder LI, Frankfurter A, Rebhun LI (1985) The distribution of tau in the mammalian central nervous system. J Cell Biol 101:1371-1378.

Bohm H, Brinkmann V, Drab M, Henske A, Kurzchalia TV (1997) Mammalian homologues of $C$. elegans PAR-1 are asymmetrically localized in epithelial cells and may influence their polarity. Curr Biol 7:603-606.

Behrmann I, Smyczek T, Heinrich PC, Schmitz-Van de Leur H, Komyod W, Giese B, Muller-Newen G, Haan S, Haan C (2004) Janus kinase (Jak) subcellular localization revisited: the exclusive membrane localization of endogenous Janus kinase 1 by cytokine receptor interaction uncovers the Jak receptor complex to be equivalent to a receptor tyrosine kinase. J Biol Chem 279:35486-35493.

Bessone S, Vidal F, Le Bouc Y, Epelbaum J, Bluet-Pajot MT, Darmon M (1999) EMK protein kinase-null mice: dwarfism and hypofertility asso- ciated with alterations in the somatotrope and prolactin pathways. Dev Biol 214:87-101

Chen YM, Wang QJ, Hu HS, Yu PC, Zhu J, Drewes G, Piwnica-Worms H, Luo ZG (2006) Microtubule affinity-regulating kinase 2 functions downstream of the PAR-3/PAR-6/atypical PKC complex in regulating hippocampal neuronal polarity. Proc Natl Acad Sci USA 103:8534-8539.

Ciani L, Krylova O, Smalley MJ, Dale TC, Salinas PC (2004) A divergent canonical WNT-signaling pathway regulates microtubule dynamics: dishevelled signals locally to stabilize microtubules. J Cell Biol 164:243-253.

Cohen D, Rodriguez-Boulan E, Musch A (2004a) Par-1 promotes a hepatic mode of apical protein trafficking in MDCK cells. Proc Natl Acad Sci USA 101:13792-13797.

Cohen D, Brennwald PJ, Rodriguez-Boulan E, Musch A (2004b) Mammalian PAR-1 determines epithelial lumen polarity by organizing the microtubule cytoskeleton. J Cell Biol 164:717-727.

Dotti CG, Sullivan CA, Banker GA (1988) The establishment of polarity by hippocampal neurons in culture. J Neurosci 8:1454-1468.

Drewes G, Ebneth A, Preuss U, Mandelkow EM, Mandelkow E (1997) MARK, a novel family of protein kinases that phosphorylate microtubuleassociated proteins and trigger microtubule disruption. Cell 89:297-308.

Elbert M, Cohen D, Musch A (2006) PAR1b promotes cell-cell adhesion and inhibits dishevelled-mediated transformation of Madin-Darby canine kidney cells. Mol Biol Cell 17:3345-3355.

Funato Y, Michiue T, Asashima M, Miki H (2006) The thioredoxin-related redox-regulating protein nucleoredoxin inhibits Wnt-beta-catenin signalling through dishevelled. Nat Cell Biol 8:501-508.

Gao FB, Kohwi M, Brenman JE, Jan LY, Jan YN (2000) Control of dendritic field formation in Drosophila: the roles of flamingo and competition between homologous neurons. Neuron 28:91-101.

Goransson O, Deak M, Wullschleger S, Morrice NA, Prescott AR, Alessi DR (2006) Regulation of the polarity kinases PAR-1/MARK by 14-3-3 interaction and phosphorylation. J Cell Sci 119:4059-4070.

Guo S, Kemphues KJ (1995) par-1, a gene required for establishing polarity in C. elegans embryos, encodes a putative Ser/Thr kinase that is asymmetrically distributed. Cell 81:611-620.

Hirokawa N, Funakoshi T, Sato-Harada R, Kanai Y (1996) Selective stabilization of tau in axons and microtubule-associated protein $2 \mathrm{C}$ in cell bodies and dendrites contributes to polarized localization of cytoskeletal proteins in mature neurons. J Cell Biol 132:667-679.

Hurov JB, Stappenbeck TS, Zmasek CM, White LS, Ranganath SH, Russell JH, Chan AC, Murphy KM, Piwnica-Worms H (2001) Immune system dysfunction and autoimmune disease in mice lacking Emk (Par-1) protein kinase. Mol Cell Biol 21:3206-3219.

Hurov JB, Watkins JL, Piwnica-Worms H (2004) Atypical PKC phosphorylates PAR-1 kinases to regulate localization and activity. Curr Biol 14:736-741.

Illenberger S, Drewes G, Trinczek B, Biernat J, Meyer HE, Olmsted JB, Mandelkow EM, Mandelkow E (1996) Phosphorylation of microtubuleassociated proteins MAP2 and MAP4 by the protein kinase p110mark. Phosphorylation sites and regulation of microtubule dynamics. J Biol Chem 271:10834-10843.

Inagaki N, Chihara K, Arimura N, Menager C, Kawano Y, Matsuo N, Nishimura T, Amano M, Kaibuchi K (2001) CRMP-2 induces axons in cultured hippocampal neurons. Nat Neurosci 4:781-782.

Itoh TJ, Hotani H (1994) Microtubule-stabilizing activity of microtubuleassociated proteins (MAPs) is due to increase in frequency of rescue in dynamic instability: shortening length decreases with binding of MAPs onto microtubules. Cell Struct Funct 19:279-290.

Kanai Y, Hirokawa N (1995) Sorting mechanisms of tau and MAP2 in neurons: suppressed axonal transit of MAP2 and locally regulated microtubule binding. Neuron 14:421-432.

Kosik KS, Finch EA (1987) MAP2 and tau segregate into dendritic and axonal domains after the elaboration of morphologically distinct neurites: an immunocytochemical study of cultured rat cerebrum. J Neurosci 7:3142-3153.

Krylova O, Messenger MJ, Salinas PC (2000) Dishevelled-1 regulates microtubule stability: a new function mediated by glycogen synthase kinase3beta. J Cell Biol 151:83-94.

Kusakabe M, Nishida E (2004) The polarity-inducing kinase Par-1 controls Xenopus gastrulation in cooperation with $14-3-3$ and aPKC. EMBO J 23:4190-4201.

Lijam N, Paylor R, McDonald MP, Crawley JN, Deng CX, Herrup K, Stevens 
KE, Maccaferri G, McBain CJ, Sussman DJ, Wynshaw-Boris A (1997) Social interaction and sensorimotor gating abnormalities in mice lacking Dvll. Cell 90:895-905.

Lizcano JM, Goransson O, Toth R, Deak M, Morrice NA, Boudeau J, Hawley SA, Udd L, Makela TP, Hardie DG, Alessi DR (2004) LKB1 is a master kinase that activates 13 kinases of the AMPK subfamily, including MARK/ PAR-1. EMBO J 23:833-843.

Malbon CC, Wang HY (2006) Dishevelled: a mobile scaffold catalyzing development. Curr Top Dev Biol 72:153-166.

Ossipova O, Dhawan S, Sokol S, Green JB (2005) Distinct PAR-1 proteins function in different branches of Wnt signaling during vertebrate development. Dev Cell 8:829-841.

Papasozomenos SC, Binder LI (1987) Phosphorylation determines two distinct species of Tau in the central nervous system. Cell Motil Cytoskeleton $8: 210-226$

Peng I, Binder LI, Black MM (1986) Biochemical and immunological analyses of cytoskeletal domains of neurons. J Cell Biol 102:252-262.

Rosso SB, Sussman D, Wynshaw-Boris A, Salinas PC (2005) Wnt signaling through Dishevelled, Rac and JNK regulates dendritic development. Nat Neurosci 8:34-42.

Sun TQ, Lu B, Feng JJ, Reinhard C, Jan YN, Fantl WJ, Williams LT (2001) PAR-1 is a Dishevelled-associated kinase and a positive regulator of Wnt signalling. Nat Cell Biol 3:628-636.

Suzuki A, Hirata M, Kamimura K, Maniwa R, Yamanaka T, Mizuno K, Kishikawa M, Hirose H, Amano Y, Izumi N, Miwa Y, Ohno S (2004) aPKC acts upstream of PAR-1b in both the establishment and maintenance of mammalian epithelial polarity. Curr Biol 14:1425-1435.

Takei Y, Teng J, Harada A, Hirokawa N (2000) Defects in axonal elongation and neuronal migration in mice with disrupted tau and maplb genes. J Cell Biol 150:989-1000.
Takenaka K, Kise Y, Miki H (2007) GSK3beta positively regulates Hedgehog signaling through Sufu in mammalian cells. Biochem Biophys Res Commun 353:501-508.

Tassan JP, Le Goff X (2004) An overview of the KIN1/PAR-1/MARK kinase family. Biol Cell 96:193-199.

Teng J, Takei Y, Harada A, Nakata T, Chen J, Hirokawa N (2001) Synergistic effects of MAP2 and MAP1B knock-out in neuronal migration, dendritic outgrowth, and microtubule organization. J Cell Biol 155:65-76.

Timm T, Li XY, Biernat J, Jiao J, Mandelkow E, Vandekerckhove J, Mandelkow EM (2003) MARKK, a Ste20-like kinase, activates the polarityinducing kinase MARK/PAR-1. EMBO J 22:5090-5101.

Uboha NV, Flajolet M, Nairn AC, Picciotto MR (2007) A calcium- and calmodulin-dependent kinase Ialpha/microtubule affinity regulating kinase 2 signaling cascade mediates calcium-dependent neurite outgrowth. J Neurosci 27:4413-4423.

Wallingford JB, Habas R (2005) The developmental biology of Dishevelled: an enigmatic protein governing cell fate and cell polarity. Development 132:4421-4436.

Wayman GA, Impey S, Marks D, Saneyoshi T, Grant WF, Derkach V, Soderling TR (2006) Activity-dependent dendritic arborization mediated by CaM-kinase I activation and enhanced CREB-dependent transcription of Wnt-2. Neuron 50:897-909.

Wiggin GR, Fawcett JP, Pawson T (2005) Polarity proteins in axon specification and synaptogenesis. Dev Cell 8:803-816.

Yu X, Malenka RC (2003) Beta-catenin is critical for dendritic morphogenesis. Nat Neurosci 6:1169-1177.

Zhang X, Zhu J, Yang GY, Wang QJ, Qian L, Chen YM, Chen F, Tao Y, Hu HS, Wang T, Luo ZG (2007) Dishevelled promotes axon differentiation by regulating atypical protein kinase C. Nat Cell Biol 9:743-754. 